\title{
Asystole during onyx embolisation of dural arteriovenous fistula: a case of trigeminal cardiac reflex
}

\author{
Zihao Song ${ }^{1}$, Yongjie $\mathrm{Ma}^{1}$, Jiewen $\mathrm{Geng}^{1}$, Peng $\mathrm{Hu}^{1}, \mathrm{Na} \mathrm{Xu}^{2}$, Hongqi Zhang ${ }^{1}$ \\ ${ }^{1}$ Department of Neurosurgery, Xuanwu Hospital, Capital Medical University, Xicheng District, Beijing, China \\ ${ }^{2}$ Department of Anaesthesiology, Xuanwu Hospital, Capital Medical University, Xicheng District, Beijing, China
}

Key words: dural arteriovenous fistula, onyx, trigeminal cardiac reflex

(Neurol Neurochir Pol 2021; 55 (4): 410-412)

\section{To the Editors}

Trigeminal cardiac reflex (TCR), hemifacial hypesthesia, hemifacial palsy, jaw pain, internal jugular vein injury and microcatheter gluing are potential complications in endovascular onyx embolisation of dural arteriovenous fistulas (DAVFs) [1]. TCR is a physiological reflex that occurs in response to stimulation of sensory branches of the trigeminal nerve, which can lead to haemodynamic instability such as bradycardia and asystole. TCR is often reported in craniofacial, ophthalmological, and skull base surgery, and less often reported in endovascular onyx embolisation. We report a case of TCR during endovascular onyx embolisation of a DAVF.
A 37-year-old male presented with a sudden severe headache without apparent cause, most pronounced in the occipital region, accompanied by nausea and vomiting. CT scan showed haemorrhage on right cerebellum, third ventricle and fourth ventricle. Further DSA examination showed a Cognard Class $\triangle \mathrm{DAVF}$ in right petroclival region. The DAVF was fed by the marginal tentorial branch of right meningohypophyseal trunk, right anterior inferior cerebellar artery, and the petrous branch of right middle meningeal artery (Fig. 1). The patient had no significant cardiac or respiratory disease. After discussion of treatment options, we decided to use transarterial onyx to embolise DAVF.

The procedure was performed under standard anaesthesia and systematic heparinisation. Anaesthesia was induced with
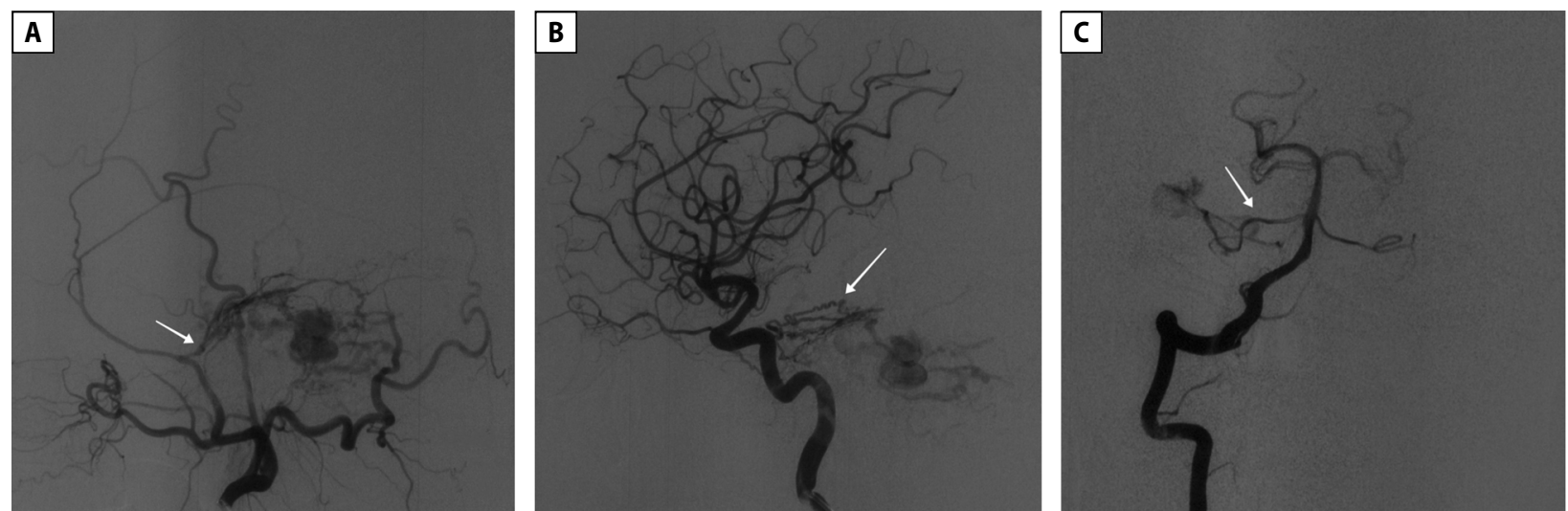

Figure 1. A. Lateral view of left external carotid artery angiogram demonstrates DAVF being fed by petrous branch of right middle meningeal artery (arrow); B. Lateral view of left internal carotid artery angiogram demonstrates DAVF being fed by marginal tentorial branch of right meningohypophyseal trunk (arrow); C. Anteroposterior view of left vertebral artery angiogram demonstrates DAVF being fed by right anterior inferior cerebellar artery (arrow)

Address for correspondence: Hongqi Zhang, Department of Neurosurgery, Xuanwu Hospital, Capital Medical University, No. 45, Changchun Street, Xicheng District, Beijing 100053, China; e-mail: xwzhanghq@163.com

Received: 23.01.2021 Accepted: 6.05.2021 Early publication date: 11.06.2021 

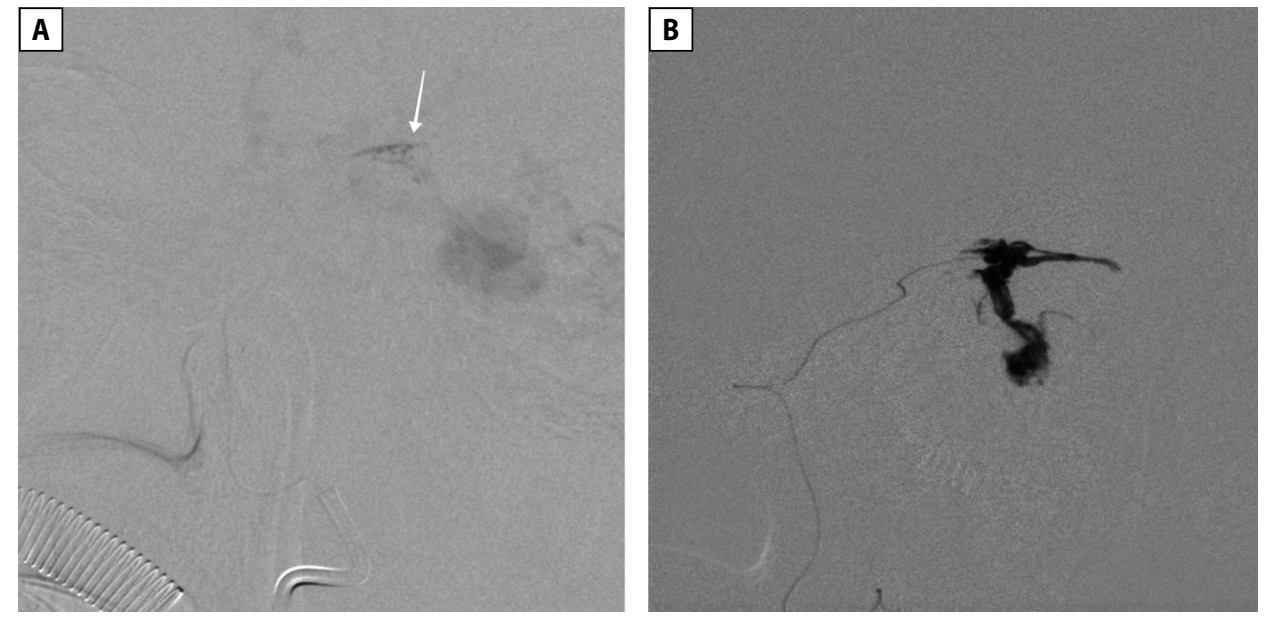

Figure 2. A. Lateral view of petrous branch of right middle meningeal artery superselective microcatheter angiogram demonstrates fistulous point (arrow); B. Lateral view of endovascular onyx injection to fistula

sufentanil $15 \mathrm{ug}$, followed by rocuronium $(0.8 \mathrm{mg} / \mathrm{kg})$ and etomidate $(0.2 \mathrm{mg} / \mathrm{kg})$. After tracheal intubation, we performed mechanical ventilation with pure oxygen. Anaesthesia was maintained with renifentanyl $(0.2 \mathrm{~g} / \mathrm{kg} / \mathrm{min})$, and additional boluses of propofol $(2 \mathrm{mg} / \mathrm{kg} / \mathrm{h})$, norepinephrine $(0.06 \mathrm{ug} / \mathrm{kg} / \mathrm{min})$, dexmedetomidine $(0.5 \mathrm{ug} / \mathrm{kg} / \mathrm{h})$ and sevoflurane $(0.4 \%)$ were administered.

After transfemoral arterial access was gained, an Envoy 6 F guide catheter (Cordis, Miami Lakes, FL, USA) was placed in the right external carotid artery. By using a road-mapping technique and selective control angiogram, a Marathon microcatheter (ev3 Neurovascular, Irvine, CA, USA) with a Synchro 2 0.010-inch microwire (Stryker Neurovascular, Fremont, CA, USA) was advanced into the petrous branch of right middle meningeal artery (Fig. 2A). Dimethyl sulfoxide (DMSO) was injected into the microcatheter without consequence. Then onyx was used to embolise the fistula (Fig. 2B). Near the completion of embolisation, the patient developed bradycardia: heart rate (HR) dropped from 52 to $32 \mathrm{bpm}$ and blood pressure (BP) from $126 / 68 \mathrm{mmHg}$ to $100 / 52 \mathrm{mmHg}$. The injection was immediately paused, and the patient's HR and BP normalised spontaneously. After discussion with the anaesthetist, onyx injection was performed again. But the patient developed asystole and BP dropped to $64 / 30 \mathrm{mmHg}$. Immediately, the injection was stopped. After intravenous injection of ephedrine and atropine and chest compressions, the patient returned to spontaneous circulation. The subsequent angiography found that the embolisation was satisfied (Fig. 3), and so the embolisation was terminated. Upon waking up from anaesthesia, the patient developed mania and was sedated with dexmedetomidine $(0.5 \mathrm{ug} / \mathrm{kg} / \mathrm{h})$ and sent to the intensive care unit (ICU) for 48 hours of close monitoring. The patient did not present bradycardia or asystole during his ICU stay, and no cardiac or respiratory symptoms were reported, except for intermittent headache. Postoperative CT showed that the

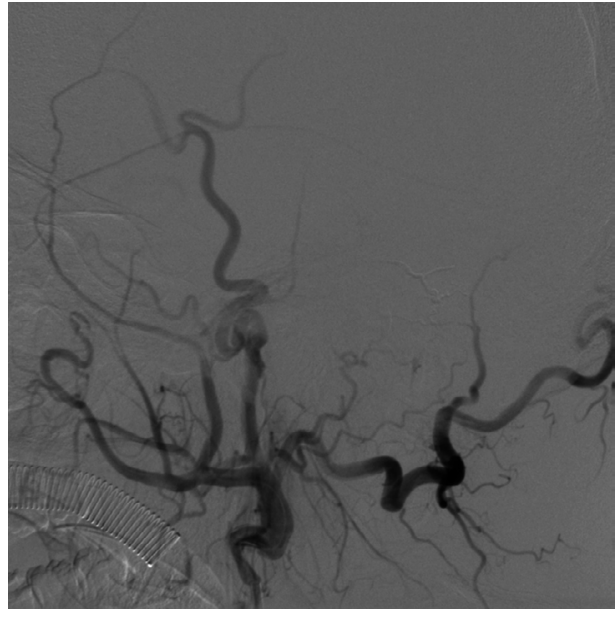

Figure 3. Lateral view of left external carotid artery angiogram demonstrates complete embolisation of fistula

bleeding was well absorbed and the patient's symptoms had improved, so he was allowed home from hospital.

This case presented herein reports bradycardia and asystole during endovascular onyx embolisation of a dural arteriovenous fistula. We consider that this response was caused by TCR, which was first described in endovascular onyx embolisation by Lv et al. [2].

Interestingly, TCR during endovascular embolisation has only been reported when using onyx. The reflex mainly occurred when transarterially injected into the middle meningeal arteries or transvenously injected into the cavernous sinus and inferior petrosal sinus. The injection of DMSO comes before the injection of onyx in order to flush the dead space of microcatheter. The incidence of TCR in endovascular onyx embolisation may be caused by neurotoxicity of DMSO or direct compression of the trigeminal nervous innervation 
of the dural mater by the formation of an onyx plug produce [3]. Wang et al. [4] thought that during transarterial onyx embolisation, the injection pressure to middle meningeal artery induced neuronal signals via the Gassarian ganglion to the sensory nucleus of the trigeminal nerve, and thus caused TCR. They also believed that neurotoxicity of DMSO on the ophthalmic nerve within the cavernous sinus, or on the trigeminal nerve innervation of the dura mater, caused TCR during transvenous onyx embolising carotid cavernous fistula. Puri et al. [5] reported a case in which TCR occurred during a pre-onyx DMSO injection. This finding suggests that it is the biochemical action of DMSO, rather than onyx or any mechanical effect of the solidified cast, which elicits the TCR [6].

In our case, TCR occurred near the end of embolisation. We think that this reflex might be due to backflow of onyx to the petrous branch, which increased the pressure of the middle meningeal artery and induced neuronal signals and elicited TCR.

Neurointerventionalists should be very cautious when conducting intravascular manipulation in the middle meningeal artery. Anaesthetists should pay close attention to heart rate changes of patients and be prepared for TCR at any time. The operation should be immediately halted once TCR occurs. Intravenous administration of anticholinergic drugs and chest compressions are efficacious.

\section{References}

1. Kawamura Y, Takigawa T, Hyodo A, et al. Transvenous embolisation via an occluded inferior petrosal sinus for cavernous sinus dural arteriovenous fistulas. Neurol Neurochir Pol. 2020; 54(6): 585-588, doi: 10.5603/PJNNS.a2020.0071, indexed in Pubmed: 33026643.

2. Lv X, Li Y, Lv M, et al. Trigeminocardiac reflex in embolization of intracranial dural arteriovenous fistula. AJNR Am J Neuroradiol. 2007; 28(9): 1769-1770, doi: 10.3174/ajnr.A0675, indexed in Pubmed: 17885228

3. LvX, Li Y, Jiang C, et al. The incidence of trigeminocardiac reflex in endovascular treatment of dural arteriovenous fistula with onyx. Interv Neuroradiol. 2010; 16(1): 59-63, doi: 10.1177/159101991001600107, indexed in Pubmed: 20377980.

4. Wang J, Wu HC, Wang WW, et al. Trigeminal cardiac reflex caused by onyx embolization of intracranial dural arteriovenous fistula. Turk Neurosurg. 2016; 26(3): 325-330, doi: 10.5137/1019-5149.JTN.800813.1, indexed in Pubmed: 27161455.

5. Puri AS, Thiex R, Zarzour H, et al. Trigeminocardiac reflex in a child during pre-Onyx DMSO injection for juvenile nasopharyngeal angiofibroma embolization. A case report. Interv Neuroradiol. 2011; 17(1): 13-16, doi: 10.1177/159101991101700103, indexed in Pubmed: 21561553.

6. Chowdhury T, Schaller B. Trigeminocardiac reflex. Academic Press 2015. 